\title{
Recent development of an UHR and ULV FE-SEM with various signal detection capabilities and its applications
}

\author{
Muto Atsushi*, Kawamata Shigeru*, Tamochi Ryuichiro*, White Sara***, \\ Nakagawa Mine* and Sato Mitsugu**
}

*Hitachi Science Systems, Ltd., 11-1, Ishikawa cho, Hitachinaka, 312-0057 Japan

**Hitachi High-Technologies Corp., 882Ichige, Hitachinaka, Ibaraki, 312-8504 Japan

***Hitachi High-Technologies America, 944 Clopper Road, Gaithersburg, MD 20878-1301, USA

A new concept field emission SEM, Hitachi's model S-4800, has been developed with a newly designed snorkel lens, enhanced mechanical/electrical stability (close to that of an in-lens FE-SEM), imaging magnifications up to $800 \mathrm{kX}$, and an 8 inch specimen handling capability. Fig. 1 shows its ultra-high resolution imaging performance with a catalyst. The 1 to $2 \mathrm{~nm}$ size Pt particles (indicated by arrows) and the carbon substrate are clearly visible.

This instrument employs a selectable signal detection system, which allows the detection of SE, $\mathrm{BSE}$, or a combination of these signals for an optimized image contrast that suits the specimen or purpose of the microscopy investigation. Fig.2 shows an example of a FIB milled transistor from a LCD. The BSE image reveals multi-layered composite information (indicated by arrows)/ On the other hand, the SE image shows the depth and inner structure of the defect area.

The S-4800 has another imaging technique, beam retarding, for achieving high-resolution at ultra-low voltages. Image resolution under this beam retarding mode has been calculated and verified as $2.0 \mathrm{~nm}$ at $500 \mathrm{~V}$ (Fig.3).

The instrument's evaluation capabilities are further broadened with various additional detectors such as YAG-BSE, EDS, and STEM. It is said that STEM imaging with a limited accelerating voltage of up to $30 \mathrm{kV}$ leads to good contrast of lower atomic number materials due to wider spread angles of transmitted electrons [1]. The STEM micrograph of carbon nanotubes (Fig.4) shows multi-walled inner structure and inner hole size clearly.

Additional applications are reported such as the combinations of EDS and STEM and the performance for a wider field of applications.

[1] Shiraga, et al. kobunshi ronbunshu. 49, 4 (1992) 353-359 


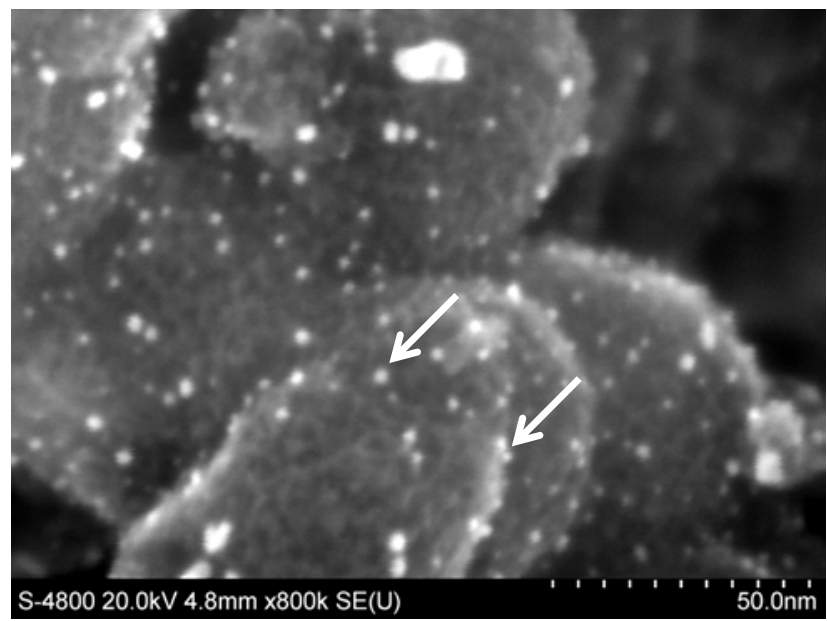

Figure 1. Ultra high resolution microscopy of Catalyst
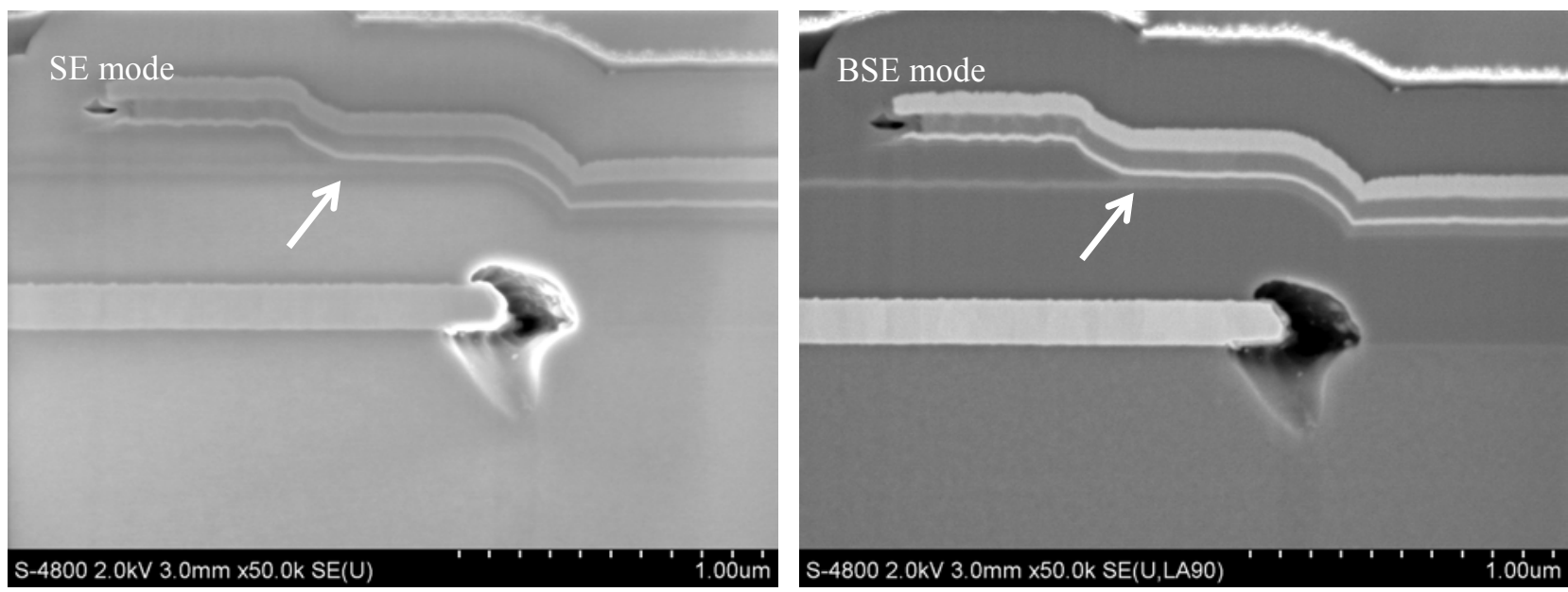

Figure 2. Cross sectional observation of FIB-milled transistor

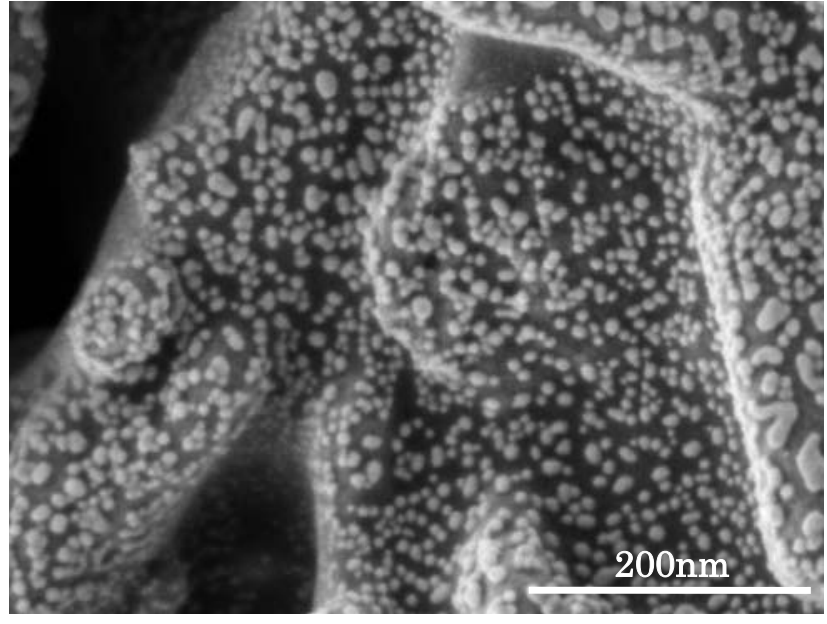

Figure 3. Resolution micrograph at 500V under retarding mode (Specimen : Au on magnetic tape)

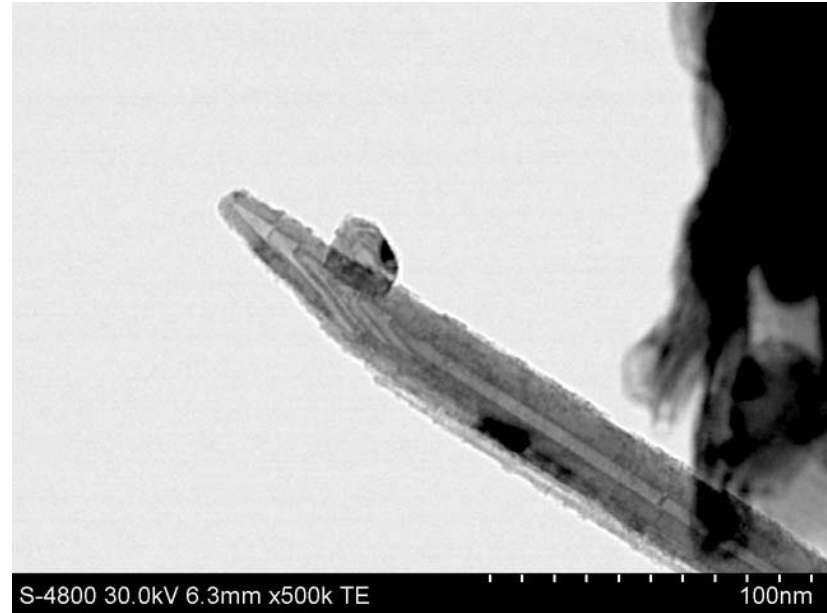

Figure 4. STEM micrograph of multi-layered carbon nanotube 